\title{
ON THE SINGULARITY OF THE EXPONENTIAL MAP ON A LIE GROUP
}

\author{
HENG-LUNG LAI ${ }^{1}$
}

\begin{abstract}
Let \&s be a connected (real or complex) Lie group with Lie algebra $G$. Define a conjugate point $g$ of \& as a point $g=\exp x$ for some $x \in G$ and $d \exp _{x}$ is a noninvertible linear map. We prove that $g \in$ (S) is a conjugate point if and only if $g=\exp x_{\lambda}$ for at least a (complex parameter) family of elements $x_{\lambda}(\lambda \in \mathbf{C})$ in $G$.
\end{abstract}

Introduction. Let $p$ be a fixed point on a connected complete Riemannian manifold $M$. Then the number of geodesics issuing from $p$ passing through a point conjugate to $p$ varies. We want to consider a similar problem for a connected Lie group \&\& with Lie algebra $G$. We call $g \in$ \&S a conjugate point if $g=\exp x$ for some $x$ in $G$ such that $d \exp _{x}$ is a noninvertible linear map. Given a conjugate point $g$, how many 1-parameter subgroups will pass through $g$ ? For a simply connected solvable Lie group, Dixmier [1] showed that there is at least a 1-parameter family of 1-parameter subgroups passing through a conjugate point. (He showed this for the complex case, and the real case can be derived from his proof.) The main purpose of this paper is to prove the following theorem by using a formula given in Helgason [2]:

THEOREM. An element $g$ of $\&$ is a conjugate point if and only if $g=\exp x_{\lambda}$ for at least a (complex parameter) family $\left\{x_{\lambda}: \lambda \in \mathbf{C}\right\}$ of elements $x_{\lambda}$ in $G$.

Proof of THEOREM. "If" part is clear.

"Only if" part: We use the formula

$$
d \exp _{x}=d\left(L_{\exp x}\right)_{e^{0}} \frac{1-\operatorname{Exp}(-\operatorname{ad} x)}{\operatorname{ad} x} \quad(x \in G),
$$

given in Helgason [2], where Exp is the exponential map of matrices, $L_{\exp x}$ is the left translation by $\exp x$ in $\&$ (which is a diffeomorphism), so that $d \exp _{x}$ is not invertible if and only if ad $x$ has some eigenvalue of the form $2 \pi \sqrt{-1} k$, where $k$ is a nonzero integer.

(i) First case: (B) is a complex Lie group.

Received by the editors February 25, 1976.

AMS (MOS) subject classifications (1970). Primary 22E10, $22 \mathrm{E} 15$.

1 This paper is a portion of the author's Ph.D. thesis. The thesis was written under the direction of Professor Morikuni Goto at the University of Pennsylvania. The author would like to take this opportunity to thank Professor Goto for his help and guidance.

() American Mathematical Society 1977 
If $g=\exp x$ is a conjugate point, we can find an eigenvector $y \in G$ of ad $x$ such that ad $x \cdot y=2 \pi \sqrt{-1} k \cdot y$. Then for any complex number $\lambda$,

$$
\begin{aligned}
& \exp x \cdot \exp \lambda y \cdot \exp -x=\exp (\operatorname{Exp} \operatorname{ad} x \cdot \lambda y) \\
& =\exp \left(\lambda y+[x, \lambda y]+\frac{1}{2}[x,[x, \lambda y]]+\cdots\right) \\
& =\exp \left(\lambda y+2 \pi \sqrt{-1} k \cdot \lambda y+\frac{1}{2}(2 \pi \sqrt{-1} k)^{2} \lambda y+\cdots\right) \\
& =\exp \left(e^{2 \pi \sqrt{-1} k} \lambda y\right)=\exp \lambda y . \\
& \therefore \exp x \cdot \exp \lambda y \cdot \exp -x \cdot \exp -\lambda y=1 \text {, }
\end{aligned}
$$

i.e.

$$
g=\exp x=\exp \lambda y \cdot \exp x \cdot \exp -\lambda y=\exp (\operatorname{Exp}(\operatorname{ad} \lambda y) \cdot x) .
$$

Since

$$
\begin{aligned}
\operatorname{ad} \lambda y \cdot x & =[\lambda y, x]=-2 \sqrt{-1} k \cdot \lambda y, \\
(\operatorname{ad} \lambda y)^{2} \cdot x & =[\lambda y,-2 \pi \sqrt{-1} k \cdot \lambda y]=0 .
\end{aligned}
$$

Therefore

$$
g=\exp x=\exp (x+2 \pi \sqrt{-1} k \cdot \lambda y) \text { for any } \lambda \text { in C. }
$$

Remark. Let $u=y+\sqrt{-1} y, v=y-\sqrt{-1} y$. Then $[x, u]=-2 \pi k \cdot v,[x$, $v]=2 \pi k \cdot u,[u, v]=0$, i.e. $\mathbf{R} x+\mathbf{R} u+\mathbf{R} v$ is the Lie algebra of the euclidean motion group of $\mathbf{R}^{2}$.

(ii) Second case: \& is a real Lie group.

Let $G_{c}$ be the complexification of $G$. For $z=u+\sqrt{-1} v \in G_{c}$ where $u, v \in G$, we denote $u-\sqrt{-1} v$ by $\bar{z}$.

Since ad $x$ is a real linear map with $2 \pi \sqrt{-1} k$ as an eigenvalue, we can find an eigenvector $z=u+\sqrt{-1} v \in G_{c}$ such that ad $x \cdot z=2 \pi \sqrt{-1} k \cdot z$. Therefore,

$$
\begin{aligned}
\operatorname{ad} x \cdot \bar{z} & =[x, u]-\sqrt{-1}[x, v]=-2 \pi k \cdot v-2 \pi k \sqrt{-1} u \\
& =-2 \pi \sqrt{-1} k(u-\sqrt{-1} v)=-2 \pi \sqrt{-1} k \cdot \bar{z}
\end{aligned}
$$

i.e. $\bar{z}$ is an eigenvector of ad $x$ with eigenvalue $-2 \pi \sqrt{-1} k$. For any $\lambda \in \mathbf{C}$,

$$
\begin{aligned}
\operatorname{ad} x \cdot(\lambda z+\overline{\lambda z}) & =2 \pi \sqrt{-1} k(\lambda z-\overline{\lambda z}), \\
(\operatorname{ad} x)^{2} \cdot(\lambda z+\overline{\lambda z}) & =2 \pi \sqrt{-1} k \cdot \operatorname{ad} x \cdot(\lambda z-\overline{\lambda z})=(2 \pi \sqrt{-1} k)^{2}(\lambda z+\overline{\lambda z}), \\
(\operatorname{ad} x)^{r} \cdot(\lambda z+\overline{\lambda z}) & =(2 \pi \sqrt{-1} k)^{r}\left(\lambda z+(-1)^{r} \cdot \overline{\lambda z}\right), \quad r=1,2,3, \ldots
\end{aligned}
$$

Hence 


$$
\begin{aligned}
\operatorname{Exp} \operatorname{ad} x \cdot( & (\lambda z+\overline{\lambda z})=\left(1+\operatorname{ad} x+\frac{(\operatorname{ad} x)^{2}}{2 !}+\cdots\right) \cdot(\lambda z+\overline{\lambda z}) \\
= & (\lambda z+\overline{\lambda z})+2 \pi \sqrt{-1} k(\lambda z-\overline{\lambda z})+\frac{(2 \pi \sqrt{-1} k)^{2}}{2 !}(\lambda z+\overline{\lambda z})+\cdots \\
= & \left(\lambda z+(2 \pi \sqrt{-1} k) \lambda z+\frac{(2 \pi \sqrt{-1} k)^{2}}{2 !} \lambda z+\frac{(2 \pi \sqrt{-1} k)^{3}}{3 !} \lambda z+\cdots\right) \\
& +\left(\overline{\lambda z}+(-2 \pi \sqrt{-1} k) \overline{\lambda z}+\frac{(-2 \pi \sqrt{-1} k)^{2}}{2 !} \overline{\lambda z}+\cdots\right) \\
= & e^{2 \pi \sqrt{-1} k} \cdot \lambda z+e^{-2 \pi \sqrt{-1} k} \cdot \overline{\lambda z}=\lambda z+\overline{\lambda z}
\end{aligned}
$$

Therefore, for $y_{\lambda}=\lambda z+\overline{\lambda z}$ in $G$, we have

$$
\exp x \cdot \exp y_{\lambda} \cdot \exp -x=\exp \left(\operatorname{Exp} \operatorname{ad} x \cdot y_{\lambda}\right)=\exp y_{\lambda} \text { for any } \lambda \text { in } \mathbf{C},
$$

i.e. $\exp -y_{\lambda} \cdot \exp x \cdot \exp y_{\lambda} \cdot \exp -x=1$.

Hence $\exp x=\exp \left(\operatorname{Ad}\left(\exp y_{\lambda}\right) \cdot x\right)$ for any $\lambda$ in $\mathbf{C} \cdot \operatorname{But} \operatorname{Ad}\left(\exp y_{\lambda}\right) \cdot x$ lies in $G$ because $y_{\lambda} \in G$.

This completes the proof. $\|$

\section{BIBLIOGRAPHY}

1. J. Dixmier, L'application exponentielle dans les groupes de Lie résolubles, Bull. Soc. Math. France 85 (1957), 113-121. MR 19, 1182.

2. S. Helgason, Differential geometry and symmetric spaces, Academic Press, New York, 1962. MR 26 \#2986.

Department of Mathematics, University of Pennsylvania, Philadelphia, Pennsylvania 19174

Current address: Department of Mathematics, National Central University, Chung-Li, Taiwan, Republic of China 